\title{
Search for New Physics Beyond the Standard Model at BaBar
}

\author{
Matthew Barrett \\ Representing the BaBar Collaboration. \\ Brunel University, Uxbridge, UB8 3PH, United Kingdom of Great Britain and Northern Ireland
}

\begin{abstract}
.
A review of selected recent $\mathrm{BaBar}$ results are presented that illustrate the ability of the experiment to search for physics beyond the standard model. The decays $B \rightarrow \tau \nu$ and $B \rightarrow s \gamma$ provide constraints on the mass of a charged Higgs. Searches for Lepton Flavour Violation could provide a clear signal for beyond the standard model physics. Babar does not observe any signal for New Physics with the current dataset.
\end{abstract}

Keywords: Beyond Standard Model BaBar

PACS: $13.20 . \mathrm{He}$

\section{INTRODUCTION}

The BaBar detector (which is described fully elsewhere [1]) is a particle detector recording events produced from electron - positron collisions at a centre of mass energy of $10.58 \mathrm{GeV}$, provided by PEP-II. This energy was selected to produce large numbers of $B$ mesons. In addition to being a " $B$-factory", PEP-II also produces large numbers of charmed mesons and tau lepton pairs.

BaBar follows the pattern of most particle physics detectors, being built from a number of subsystems comprising layers around the interaction point. The innermost Silicon Vertex Tracker, is surrounded by the Drift Chamber. These systems provide primary tracking information for the experiment. The next subsystem is the Detector of Internally Reflected Cherenkov Radiation, which is used for particle identification. An Electromagnetic calorimeter provides all the calorimetry for the detector. A 1.5T magnet provides the prerequisite magnetic field, and the Instrumented Flux Return of this magnet also provides the muon identification.

The Babar detector had recorded an integrated luminosity of $391 \mathrm{fb}^{-1}$ as of the end of June 2007. The actual luminosity used in each of the analyses covered in this document are detailed in the discussion of the analysis.

\section{THE DECAY $B \rightarrow \tau v$}

In the standard model the Branching fraction for the process $B \rightarrow \ell v$ is given by:

$$
\mathscr{B}(B \rightarrow \ell v)=\frac{G_{F}^{2} m_{B}}{8 \pi} m_{\ell}^{2}\left(1-\frac{m_{\ell}^{2}}{m_{B}^{2}}\right)^{2} f_{B}^{2}\left|V_{u b}\right|^{2} \tau_{B}
$$


where $G_{F}$ is the Fermi constant, $m_{B}$ is the $B$ meson mass, $m_{\ell}$ is the lepton mass, $f_{B}$ is the $B$ meson decay constant, $V_{u b}$ is a CKM matrix element, and $\tau_{B}$ is the $B$ meson mean lifetime. Helicity suppression leads to a ratio between the $\tau: \mu$ : e modes of $1: 5 \times 10^{-3}$ $: 10^{-7}$, thus $B \rightarrow \tau \nu$ is the most accessible mode to search for purely leptonic decays of the $B$ meson. The Standard Model prediction is $\mathscr{B}(B \rightarrow \tau v) \sim 1.6 \times 10^{-4}$.

Beyond the standard model, there can be an additional contribution from a charged Higgs boson, replacing the $W$ Boson. In the Two Higgs Doublet Model (2HDM) [2] the branching fraction is modified in the following way:

$$
\mathscr{B}^{2 H D M}=\mathscr{B}^{S M}\left(1-\frac{m_{B}^{2} \tan ^{2} \beta}{m_{H^{ \pm}}^{2}}\right)^{2}
$$

where $m_{H}$ is the mass of the charged Higgs boson, and $\tan \beta$ is the ratio of the vacuum expectation values of the Higgs doublets.

The analysis of this decay is experimentally challenging due to the presence of two or three neutrinos in the final state. This leads to a lack of kinematic constraints on the signal $B$ meson. The other $B$ meson in the decay is fully reconstructed, and known as $B_{\text {reco }}$. The properties of the signal $B$ meson can thus be inferred from the properties of $B_{\text {reco }}$. The type of decays used to reconstruct this particle are referred to as the "tag". BaBar carries out analyses of this mode using two statistically independent tags hadronic tags consist the decay $B \rightarrow D^{(*)} X$, where $X$ is up to 6 light hadrons $\left(\pi^{ \pm}, \pi^{0}\right.$, $K^{ \pm}$, or $\left.K_{S}^{0}\right)$. Semileptonic tags reconstruct the decay $B \rightarrow D \ell v X$ where only the neutrino is missing, and $X$ is a $\pi^{0}, \gamma$ or nothing.

The reconstruction of the $\tau$ lepton is done for four modes: $\tau \rightarrow e v v, \tau \rightarrow \mu \nu v$, $\tau \rightarrow \pi \nu, \tau \rightarrow \rho(\pi \pi) \nu, \tau \rightarrow a_{1}(\pi \pi \pi) v$. These modes make up about $70 \%$ of the total $\tau$ lepton branching fraction.

The most important variable for distinguishing signal from background is $E_{\text {Extra }}$, which is the sum of all the energy in the calorimeter that is not attributed to one of the reconstructed particles in the event. A cut on this variable of less than approximately $0.3 \mathrm{GeV}$ (mode dependent), forms a signal region in which the expected number of background events is calculated using studies of Monte Carlo (MC) and off-resonance data (data taken at a centre of mass energy $40 \mathrm{MeV}$ lower than the nominal value, which is below the threshold for the production of $B$ mesons).

Each $\mathrm{BaBar}$ analysis performs a maximum likelihood fit to extract the yield of events in the signal region for each mode, incorporating probability density functions (PDFs) for signal events, and the expected background. This likelihood fit can be used to extract the branching fraction measurement.

For the semileptonic tags analysis[3] the preliminary branching fraction is $\mathrm{B}(B \rightarrow$ $\tau v)=(0.9 \pm 0.6$ (stat) \pm 0.1 (syst) $) \times 10^{-4}$, where the first error is statistical and the second is systematic. Since this measurement does not have a high statistical significance, an upper limit is calculated. The upper limit is $1.7 \times 10^{-4}$ at a $90 \%$ confidence level.

The hadronic analysis [4] reports a preliminary branching fraction of $\mathrm{B}=\left(1.8_{0.9}^{1.0}\right.$ (stat.+bkg.) \pm 0.3 (syst.) $) \times 10^{-4}$, where the first error incorporates the statistical and background systematics, and the second error is the remaining systematics. The $90 \%$ confidence level upper limit on this measurement is $B<3.4 \times 10^{-4}$. Both of these 
analyses used a luminosity corresponding to 383 million $B \bar{B}$ meson pairs.

Since the two measurements for this decay are statistically independent, they can be combined. This is achieved by extending the maximum likelihood fit to encompass all the yields. This results in an overall branching fraction measurement of $\mathscr{B}(B \rightarrow$ $\tau v)=\left(1.20_{-0.38}^{+0.40}(\text { stat } .)_{-0.30}^{+0.29}(\right.$ bkgsyst.$) \pm 0.22($ syst.$\left.)\right) \times 10^{-4}$. The Belle collaboration has published a result for this mode of $\mathscr{B}(B \rightarrow \tau v)=\left(1.79_{-0.49}^{+0.56}(\text { stat } .)_{-0.46}^{+0.39}(\right.$ syst.$\left.)\right) \times$ $10^{-4}[5]$.

The branching fraction result can also be used to constrain new physics involving charged a Higgs, and combined with constraints from the analysis of $B \rightarrow s \gamma$.

\section{THE DECAY $B \rightarrow s \gamma$}

The decay $B \rightarrow s \gamma$ is a flavour changing neutral current, which is forbidden at tree level in the SM. It proceeds via a penguin loop, involving a $W$ boson, and $u$-type quark, which is dominated by the contribution from the $t$ quark. Beyond the SM the $W$ boson could be replaced with a charged Higgs boson, or the $W$ boson and quark could be replaced by supersymmetric chargino and squark. In rare decays proceeding through penguin loops, contributions from new physics could prove significant.

There are two methods for studying the decay $B \rightarrow s \gamma$ at BaBar - Fully Inclusive, and Sum of Exclusive. The fully inclusive method involves only reconstructing the photon, this is theoretically clean, but there are high backgrounds. The sum of Exclusive method involves reconstructing a subset of final states arising from the $s$ quark $-X_{s}$. This method has a lower background, but there is an additional $X_{s}$ fragmentation systematic, and missing $X_{S}$ modes. These proceedings discuss a fully inclusive analysis.

The signature for this decay is an isolated high energy photon. Any photons compatible with decays from $\pi^{0}, \eta$, or $\rho$ decays are vetoed. This analysis is carried out using the recoil method described for the aforementioned $B \rightarrow \tau v$ analysis, and uses hadronic tags. It was carried out using $210 \mathrm{fb}^{-1}$ of data.

The SM theoretical prediction for $\mathscr{B}\left(B \rightarrow s \gamma\right.$ is $(3.16 \pm 0.23) \times 10^{-4}$ [6] for photon energy $E_{\gamma}>1.6 \mathrm{GeV}$. The BaBar preliminary measurement is $(3.66 \pm 0.85 \pm 0.59) \times$ $10^{-4}$ for $E_{\gamma}>1.9 \mathrm{GeV}$ - using an extrapolation technique [7], this gives a preliminary extrapolation of $(3.91 \pm 0.91 \pm 0.63) \times 10^{-4}$ for $E_{\gamma}>1.6 \mathrm{GeV}$. The experimental errors are statistical and systematic respectively. There is no significant discrepancy between the theoretical and experimental measurements, given the large errors on the measurement.

The branching fraction measurements from $B \rightarrow \tau \nu$ and $B \rightarrow s \gamma$ give complementary exclusions on the mass of a charged Higgs in the $m_{H}-\tan \beta$ plane.

\section{LEPTON FLAVOUR VIOLATION}

Lepton Flavour Violation (LFV) only enters the standard model through neutrino oscillations. However the Branching fraction of LFV $\tau$ decays due to neutrino oscillation is predicted to be $\sim \mathscr{O}\left(10^{-54}\right)$, therefore any observation of LFV decays would be a signal 
TABLE 1. Branching fraction upper limits of LFV $\tau$ decays at $90 \%$ confidence level.

\begin{tabular}{|c|c|c|c|c|c|c|}
\hline & \multicolumn{3}{|c|}{ BaBar } & \multicolumn{3}{|c|}{ Belle } \\
\hline Mode & $\mathscr{B}_{U L}\left(10^{-7}\right)$ & $\mathscr{L}\left(\mathrm{fb}^{-1}\right)$ & Reference & $\mathscr{B}_{U L}\left(10^{-7}\right)$ & $\mathscr{L}\left(\mathrm{fb}^{-1}\right)$ & Reference \\
\hline$\tau \rightarrow e \gamma$ & 1.1 & 232.2 & [8] & 1.2 & 535.0 & [9] \\
\hline$\tau \rightarrow \mu \gamma$ & 0.7 & 232.2 & [10] & 0.5 & 535.0 & [9] \\
\hline$\tau \rightarrow \ell \pi^{0} \eta \eta^{\prime}$ & $1.1-1.6$ & 339.0 & [11] & $0.8-1.2$ & 401.0 & [12] \\
\hline$\tau \rightarrow \ell \ell \ell$ & $1-3$ & 91.5 & [13] & $2-4$ & 87.1 & [14] \\
\hline$\tau \rightarrow \ell h h$ & $1-5$ & 221.4 & [15] & $2-8$ & 158.0 & [16] \\
\hline
\end{tabular}

of new physics. New physics contributions, involving Higgs bosons or sparticles could lead to measurable LFV branching fractions in $\tau$ decays.

There are active searches for LFV in the decay modes: $\tau \rightarrow \ell \gamma$, and $\tau \rightarrow \ell \ell \ell$. SM backgrounds in the search for $\tau \rightarrow \ell \gamma$ include $\tau \rightarrow \ell v_{\ell} v_{\tau} \gamma$ and $e^{+} e^{-} \rightarrow \mu^{+} \mu^{-} \gamma$. Bhabha and di-muon events are the major SM backgrounds for $\tau \rightarrow \ell \ell \ell$. Unlike most $\tau$ decays these LFV modes have no neutrinos in the £nal state, and thus have no missing momentum. Current limits on LFV in $\tau$ decays are $\sim \mathscr{O}\left(10^{-7}\right)$, and BaBar measurements, together with Belle measurements are shown in Table 1.

\section{SUMMARY}

BaBar is able to search for new physics beyond the standard model through analysis of rare decay modes. BaBar is continuing this search, and will continue taking data until September 2008. So far no clear signal for beyond the SM physics has been observed, but the full $\mathrm{BaBar}$ dataset will have increased sensitivity to rare decays. Indirect searches for new physics are complementary to direct searches, such as those in the proposed LHC physics programme, and will still offer an exciting opportunity to search for new physics, even if direct searches find no new beyond the SM particles.

\section{REFERENCES}

1. B. Aubert et. al. (BaBar Collaboration) Nucl. Instrum. Meth., A479:1-116, 2002.

2. W.S.Hou PRD 482342 (1993)

3. arXiv:0705.1820v1 [hep-ex]

4. arXiv:0706.2030v1 [hep-ex]

5. K. Ikado et. al. (Belle Collaboration) PRL 97, 251802 (2006)

6. M. Misiak et. al. PRL98.022002(2007)

7. arXiv:hep-ph/0507253v3

8. B. Aubert et. al. (BaBar Collaboration) PRL96(2006)41801

9. K. Abe et. al. (Belle Collaboration) hep-ex/0609049

10. B. Aubert et. al. (BaBar Collaboration) PRL95(2005)41802

11. B. Aubert et. al. (BaBar Collaboration) PRL(2007)061803

12. K. Abe et. al. (Belle Collaboration) hep-ex/0609013

13. B. Aubert et. al. (BaBar Collaboration) PRL92(2004)121801

14. K. Abe et. al. (Belle Collaboration) PLB589(2004)103

15. B. Aubert et. al. (BaBar Collaboration) PRL95(2005)191801

16. K. Abe et. al. (Belle Collaboration) PLB640(2006)138 